\title{
Collision Warning Algorithm for Passage of an Uncontrolled Road Intersection
}

\author{
Janeks Ahrems \\ Department of Transport Electronics and Telematics \\ Riga Technical University \\ Riga, Latvia \\ janek@elikss.lv
}

\begin{abstract}
Road traffic safety is one of the priorities in development of intelligent transport systems. Collision warning devices, which operate on the basis of integrated use of satellite navigation and vehicle-to-vehicle communication ( $22 \mathrm{~V})$, are intended to supplement the existing driver-assisting systems. The present article proposes an algorithm of safe passage of uncontrolled road intersection using information obtained by means of the GPS and V2V.
\end{abstract}

Keywords-collision warning algorithm; intelligent transport systems; vehicle-to-vehicle;

\section{INTRODUCTION}

Despite the rapid qualitative and quantitative development of automobile transport throughout the world, the issue of ensuring road traffic safety is the most topical in today's context. Design of intelligent transport system as a set of measures for road traffic safety optimization, is the most promising area. Recent progress in the field of communication and navigation systems and the development of information technologies have helped create devices ensuring safe and comfortable driving conditions for a motor vehicle driver.

Of the whole variety of driving situations, passage of an uncontrolled road intersection is the most dangerous. Besides, poor visibility due to roadside structures, unfavourable weather conditions, and the driver's inadequate judgement of a distance to an approaching vehicle and of its speed may increase the risk of a road accident.

Intersection collision prevention algorithms are based on exchange of information about the location, speed, course angle and other parameters of all the participants of the traffic movement with the aim of its further analysis. Additionally, an intersection configuration may be used, which is either specified in the digital map or transferred by the road side equipment. The algorithms, which are presented in [1] and [2], provide for the use of road infrastructure. The given algorithms require the use of a set of road traffic sensors, infrared transmitters and the V2I (vehicle-to-infrastructure) communication system. Algorithm which is presented in [3] is based on GPS and pre-collected information from car's sensors. The collision warning algorithm, as described in [4], is based on forecasting of a minimum distance between the moving transport vehicles and its comparison with the predefined safe distance. However, the said algorithm does not take into account the overall sizes of motor vehicles, which, under dense traffic conditions, prevents optimal use of an intersection. The applicability of vehicle-to-vehicle communication based on a $5.8 \mathrm{GHz}$ DSRC (Dedicated Short Range Communication) system for information exchange in a built-up area is evaluated in [5]. This article suggest further development of the intersection collision warning algorithm based on information exchange by means of vehicle-to-vehicle communication.

\section{INFORMATION RECEIPT AND EXCHANGE}

Data on a vehicle's location, speed, and movement direction is obtained by means of the GPS. The GPS information update rate depends on the employed receiver and lies within the limits of $0.1-1.0 \mathrm{~Hz}$. Location information received by means of a satellite system, i.e. geographical latitude, longitude and altitude, is expressed in the format of WGS-84. Accuracy of detecting location coordinates by a local GPS receiver cannot be better than 10-15 m, depending on circumstances. On this basis, for the purpose of solving the task of accident alert, a real-time GPS featuring differential correction of navigation parameters - the so-called DGPS should be used, which provides detection accuracy of approximately 0.5 meter.

To solve the problem of collision warning on a plane, one must accomplish a successive transition to the ENU (eastnorth-up) coordinate system as described in [6]. The zero (origin) point is taken to be the location of the antenna of a GPS-receiver.

Exchange of information required for the algorithm is achieved by means a vehicle-to-vehicle (V2V) communication system. Physical and data link layers for such exchange are regulated by IEEE $802.11 \mathrm{p}$ standard. Message contents, intervals and priority are defined by Standard SAE J2735.

For transmission of information about vehicle coordinates in terms of the SAE J2735 Standard, the so-called BSM (Basic Safety Message) is used [7]. The message is transmitted in a broadcast mode at the interval of $100 \mathrm{~ms}$. In addition to the information about the vehicle's location, the message contains data on the vehicle's speed, course angle north-up, status of the brake lights, overall sizes of the vehicle, etc. 


\section{CAR MODEL}

For the purposes of establishing a safety criterion, it is worthwhile plotting a vehicle not as a single point coinciding with the GPS antenna location, but as a certain rectangle representing not just the overall sizes of a vehicle but also a certain predefined safety zone, which will determine the minimum allowable distance between vehicles as shown in Fig.1.

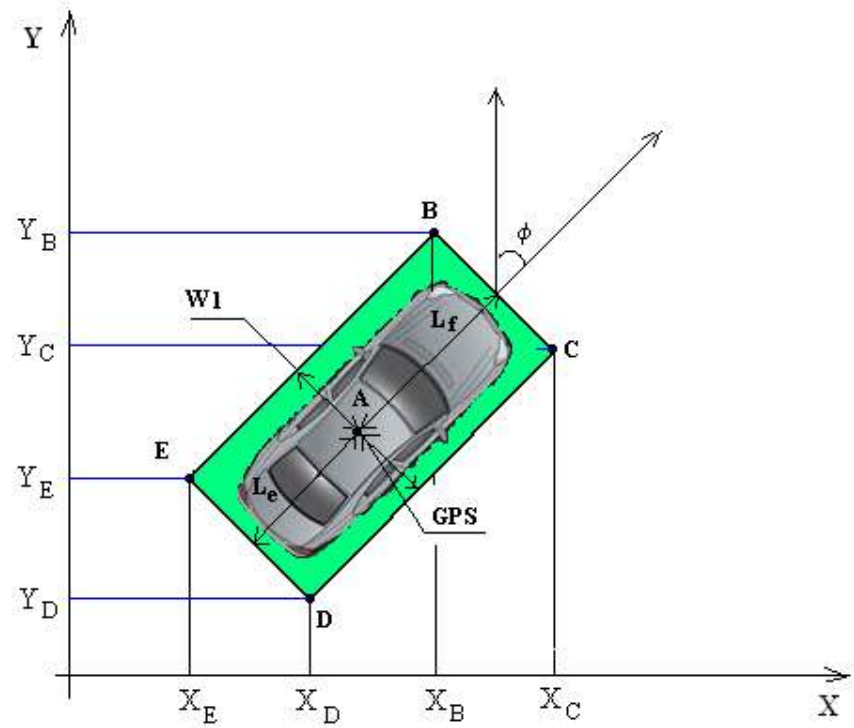

Fig. 1. Car model with predefined safety zone

Here, Point $\mathrm{A}$ is the location of a GPS antenna, $\mathrm{L}_{\mathrm{f}}-$ distance to the front limit of a safety zone, $\mathrm{L}_{\mathrm{e}}$ - distance to the end limit of a safety zone, $\mathrm{W}_{\mathrm{r}}$ - distance to the right-side limit of the safety zone, and $\mathrm{W}_{1}$ - distance to the left-side limit of the safety zone. Vehicle orientation on a plane is determined by the course angle $\varphi$, i.e. one of the parameters received from the GPS.

The coordinates of outer extremities are established using the following ratios (1) and (2):

$$
\begin{gathered}
\left\{\begin{array}{l}
X_{B}=X_{A}+L_{f} \sin \varphi-W_{l} \cos \varphi \\
X_{C}=X_{A}+L_{f} \sin \varphi+W_{r} \cos \varphi \\
X_{D}=X_{A}-L_{e} \sin \varphi+W_{r} \cos \varphi \\
X_{E}=X_{A}-L_{e} \sin \varphi-W_{l} \cos \varphi
\end{array}\right. \\
\left\{\begin{array}{l}
Y_{B}=Y_{A}+L_{f} \cos \varphi+W_{l} \sin \varphi \\
Y_{C}=Y_{A}+L_{f} \cos \varphi-W_{l} \sin \varphi \\
Y_{D}=Y_{A}-L_{e} \cos \varphi-W_{r} \sin \varphi \\
Y_{E}=Y_{A}-L_{e} \cos \varphi+W_{r} \sin \varphi
\end{array}\right.
\end{gathered}
$$

where $\mathrm{X}_{\mathrm{A}}$ and $\mathrm{Y}_{\mathrm{A}}$ are the GPS antenna location coordinates.

The resultant geometrical model of a transport vehicle will be used in solving the problem of prevention of collisions. It should be noted, however, that the given model takes no note of slippage or skidding, i.e., the vehicle's centre line coincides with the movement direction.

\section{ESTABLISHMENT OF SAFETY CRITERION}

By applying the above model to all motor vehicles participating in the traffic movement, one may establish an overall traffic safety criterion. Mutual arrangement of two moving vehicles is shown in Fig.2.

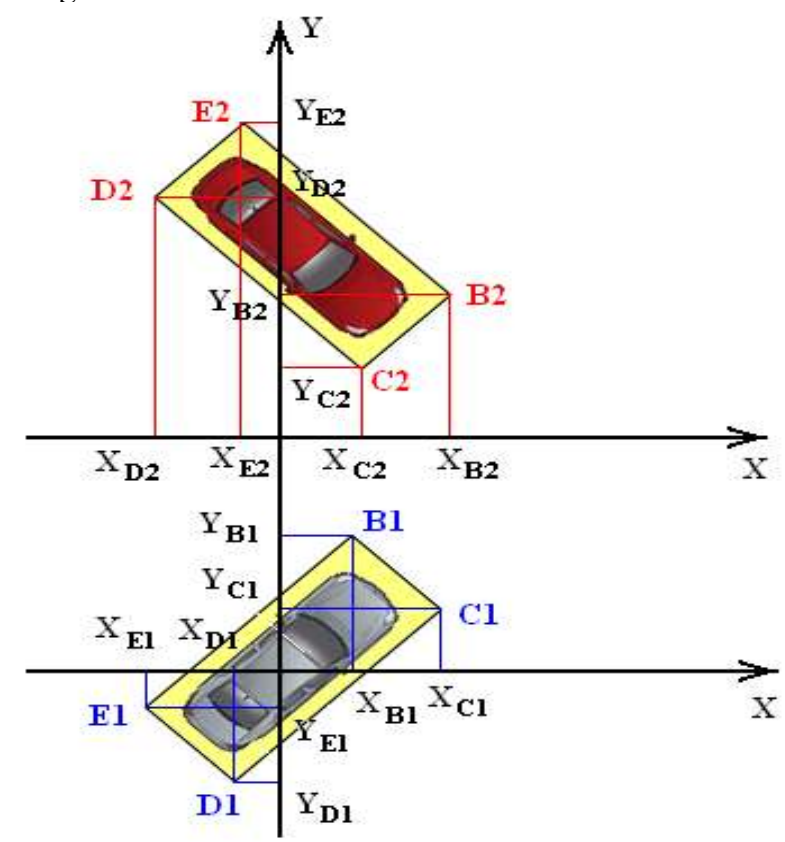

Fig. 2. Mutual arrangement of two moving vehicles

The main prerequisite for prevention of motor vehicle collisions is that there must be no simultaneous overlapping of the projections of two vehicles along the axes $\mathrm{X}$ and $\mathrm{Y}$ as shown in Fig.3.
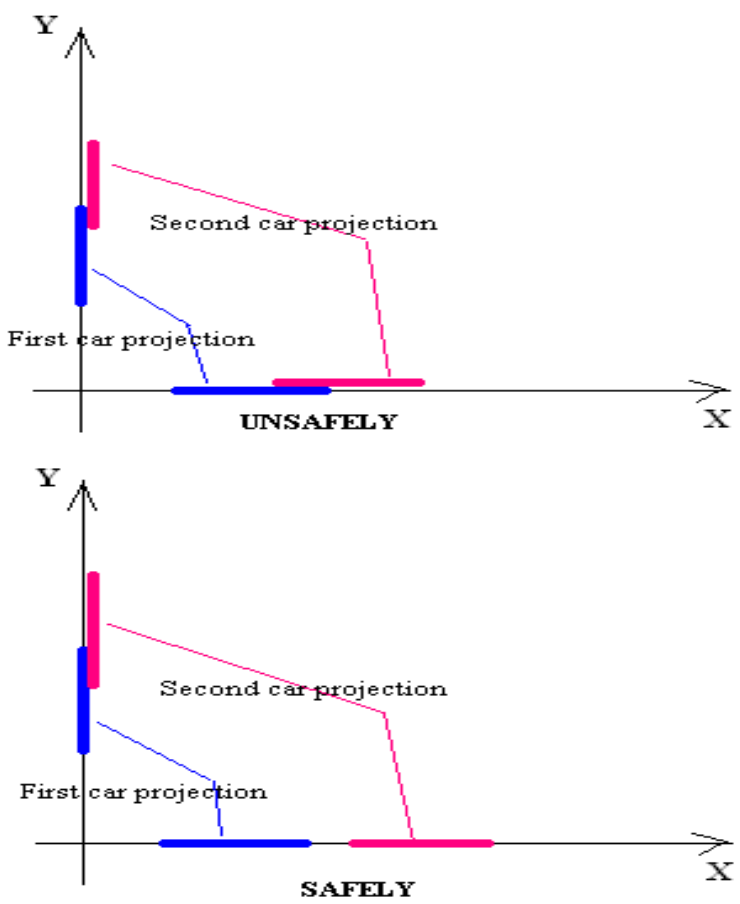

Fig. 3. Two cars projection's overlapping 
For mathematical representation of this prerequisite, one must formulate the $S_{X}(t)$ and $S_{Y}(t)$ functions, which determine the overlapping of the vehicles' projections along the axes $X$ and $\mathrm{Y}$ respectively (3):

$$
\left\{\begin{array}{l}
S_{X}(t)=L_{X}-\left(L 1_{X}+L 2_{X}\right) \\
S_{Y}(t)=L_{Y}-\left(L 1_{Y}+L 2_{Y}\right)
\end{array}\right.
$$

Here, $\mathrm{L}_{\mathrm{X}}$ and $\mathrm{L}_{\mathrm{Y}}$ are established as follows (4):

$$
\left\{\begin{array}{l}
L_{X}=[M A X(X)-\operatorname{MIN}(X)] \\
L_{Y}=[\operatorname{MAX}(Y)-\operatorname{MIN}(Y)]
\end{array},\right.
$$

where $X=\left\{X_{B 1}, X_{C 1}, X_{D 1}, X_{E 1}, X_{B 2}, X_{C 2}, X_{D 2}, X_{E 2}\right\}$, $Y=\left\{Y_{B 1}, Y_{C 1}, Y_{D 1}, Y_{E 1}, Y_{B 2}, Y_{C 2}, Y_{D 2}, Y_{E 2}\right\}$. $\mathrm{L} 1_{X}, \mathrm{~L} 2_{X}$ and $\mathrm{L} 1_{Y}, \mathrm{~L} 2_{\mathrm{Y}}$ respectively (5):

$$
\left\{\begin{array}{l}
L 1_{X}=\operatorname{MAX}(X 1)-\operatorname{MIN}(X 1) \\
L 1_{Y}=\operatorname{MAX}(Y 1)-\operatorname{MIN}(Y 1) \\
L 2_{X}=\operatorname{MAX}(X 2)-\operatorname{MIN}(X 2) \\
L 2_{Y}=\operatorname{MAX}(Y 2)-\operatorname{MIN}(Y 2)
\end{array},\right.
$$

where $X 1=\left\{X_{B 1}, X_{C 1}, X_{D 1}, X_{E 1}\right\}, Y 1=\left\{Y_{B 1}, Y_{C 1}, Y_{D 1}, Y_{E 1}\right\}$, $X 2=\left\{X_{B 2}, X_{C 2}, X_{D 2}, X_{E 2}\right\}, Y 2=\left\{Y_{B 2}, Y_{C 2}, Y_{D 2}, Y_{E 2}\right\}$.

An example of projections to the axis $X$ and the values established in (4) and (5) are shown in Fig.4.

As a result, the $S_{X}(t)$ and $S_{Y}(t)$ functions shall be specified as follows (6):

$$
\left\{\begin{array}{l}
S_{X}(t)=[\operatorname{MAX}(X)-\operatorname{MIN}(X)]- \\
-[(\operatorname{MAX}(X 1)-\operatorname{MIN}(X 1))+(\operatorname{MAX}(X 2)-\operatorname{MIN}(X 2))] \\
S_{Y}(t)=[M A X(Y)-M I N(Y)]- \\
-[(M A X(Y 1)-\operatorname{MIN}(Y 1))+(\operatorname{MAX}(Y 2)-\operatorname{MIN}(Y 2))]
\end{array}\right.
$$

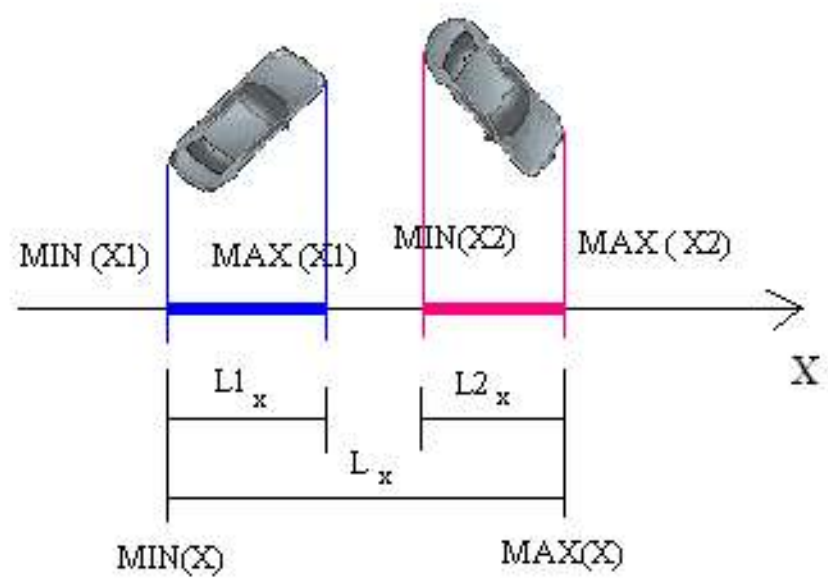

Fig. 4. Example of projections to the axis $\mathrm{X}$
Thus, having analyzed the time-dependent behaviour of the $S_{X}$ $(\mathrm{t})$ and $\mathrm{S}_{\mathrm{Y}}(\mathrm{t})$ functions, we conclude that the traffic movement is safe. That is to say, whenever the prerequisite is met (7):

$$
S_{X}(t)>0 \text { OR } S_{Y}(t)>0,
$$

the traffic movement may be accepted as safe at any point of time. The $S_{X}(t)$ and $S_{Y}(t)$ functions must be analysed pairwise for all transport vehicles participating in the road traffic (the ones we have information about).

\section{Simulation OF A DRIVING SituAtion}

There are several possible scenarios crossing the intersection: movement with a stopping at the border of intersection, turn right, turn left, etc. In this article we will consider the situation crossing the intersection without stopping in the forward direction.

Fig. 5 presents a typical driving situation where one's (own) Vehicle No 1 is approaching an uncontrolled intersection to cross it.

To evaluate the safety of the intersection passage, one must formulate an equation of motion for one's own vehicle (8):

$$
\left\{\begin{array}{l}
X_{1}=v_{1 x} t+a_{1 x} t^{2} / 2 \\
Y_{1}=v_{1 y} t+a_{1 y} t^{2} / 2
\end{array}\right.
$$

For other participants, the equation of motion will be as follows (9):

$$
\left\{\begin{array}{l}
X_{2}=X_{20}+v_{2 x} t+a_{2 x} t^{2} / 2 \\
Y_{2}=Y_{20}+v_{2 y} t+a_{2 y} t^{2} / 2 \\
X_{3}=X_{30}+v_{3 x} t+a_{3 x} t^{2} / 2 \\
Y_{3}=Y_{30}+v_{3 y} t+a_{3 y} t^{2} / 2
\end{array}\right.
$$

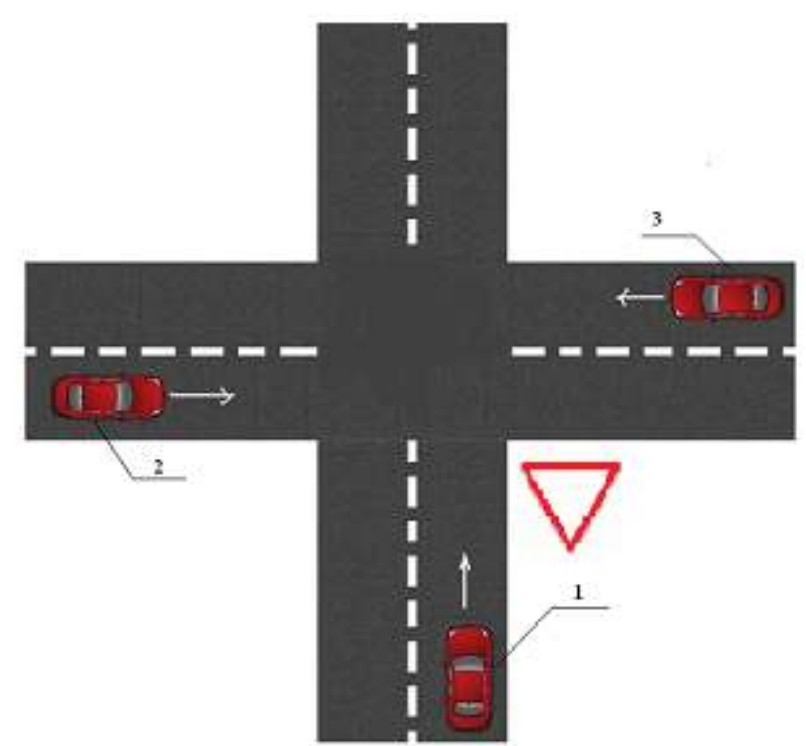

Fig. 5. Typical driving situation 
The graphs in Fig. 6 reflect the dependence of the $S_{X}(t)$ and $\mathrm{S}_{\mathrm{Y}}(\mathrm{t})$ functions pairwise for Vehicles No 1 and No 2, and Vehicles No 1 and No 3.

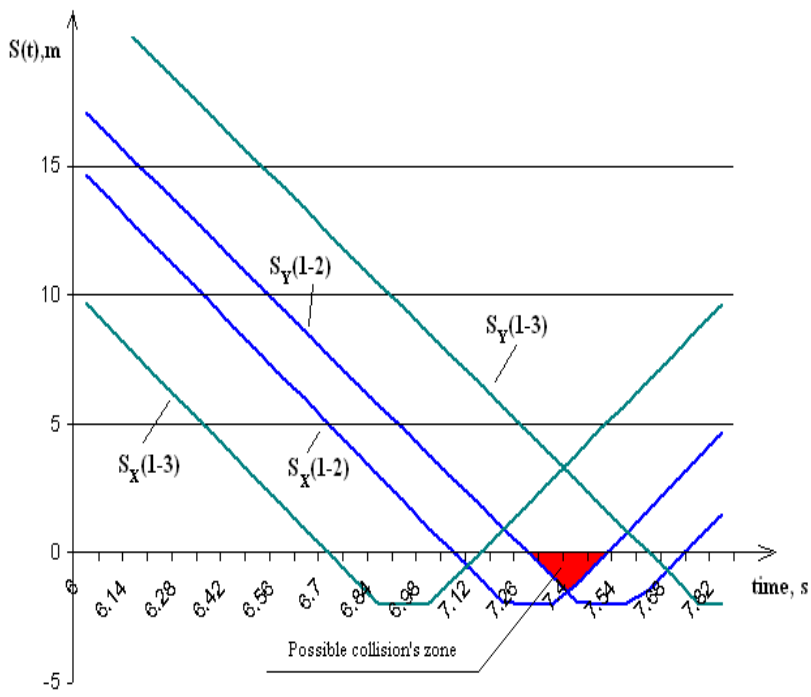

Fig. 6. Dependence of the $\mathrm{S}_{\mathrm{X}}(\mathrm{t})$ and $\mathrm{S}_{\mathrm{Y}}(\mathrm{t})$ functions from the time

The parameters of all the vehicles are given in Table 1.

TABLE I. VEHICLES' PARAMETERS

\begin{tabular}{|c|c|c|c|c|c|c|c|}
\hline $\begin{array}{c}\text { Car } \\
\text { No }\end{array}$ & Lf,m & Le,m & Wl,m & Wr,m & X0,m & Y0,m & V,m/s \\
\hline 1 & 1 & 3 & 1 & 1 & 0 & 0 & 13.5 \\
\hline 2 & 1 & 3 & 1 & 1 & -100 & 100 & 13.89 \\
\hline 3 & 1 & 3 & 1 & 1 & 95 & 105 & 13.89 \\
\hline
\end{tabular}

The graphs show it quite clearly that, the cars' parameters being as aforesaid, there is a strong likelihood of a collision between Vehicle No 1 (one's own) and Vehicle No 2. According to the Road Traffic Rules, the main operation for prevention of a danger situation on the road is braking. Therefore, the warning system must suggest that the driver should slow down the car speed to such level as specified by the established safety criterion as shown in Fig.7.

Slowing down the speed of one's car to $13.05 \mathrm{~m} / \mathrm{s}$ will ensure completely safe passage of the intersection.

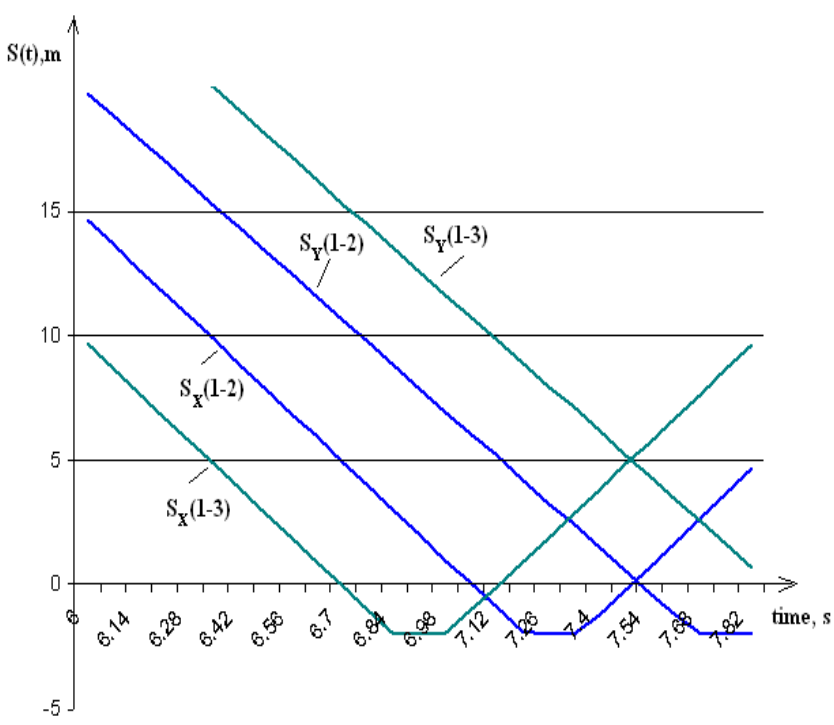

Fig. 7. Dependence of the $S_{X}(t)$ and $S_{Y}(t)$ with $v 1=13.05 \mathrm{~m} / \mathrm{s}$

\section{CONCLUSION}

The given work has provided a safety criterion for safe passage of an uncontrolled road intersection based on information about the car sizes and vehicle movement parameters. Further research will make use of the specified collision warning algorithm for passing an intersection from halting, in forward movement and with turns.

\section{REFERENCES}

[1] N.Tsukada, K. Yamada., S. Takahashi, “ Development of Driving Safety Support Systems for Non-right-of-way Vehicles at Unsignalized Intersection," $19^{\text {th }}$ ITS World Congress, Vienna, Austria, 22/26 October 2012.

[2] F.Basma, Y.Tachwali, H. H Refai,'Intersection Collision Avoidance System Using Infrastructure Communication,"1 $14^{\text {th }}$ International IEEE Conference on Intelligent Transportation Systems, Washington, USA, October 5-7, 2011.

[3] M.R.Hafner, D. Cunningham, L.Caminiti, D. Del Vecchio,"Cooperative Collision Avoidance at Intersections: Algorithms and Experiments," IEEE Transactions on ITS, Vol.14, No.3, pp. 1162-1175, 2013.

[4] J.Ahrems, "Algorithm for determination of safety intersections," In Proc. 13th Biennial Baltic Electronics Conference (BEC2012), Tallinn, Estonia, pp. 175-178, 2012.

[5] J.Ahrems, "Appraisal of Feasibility of Using Vehicle-To-Vehicle Communications for Safe Passage of Unsignalled Road Intersection under Varying Conditions," IFAC Proceedings Volumes (IFACPapersOnline). Vol.12, Part 1,pp. 84-89, 2013.

[6] C. W. Hsu, C. N. Liang, L. Y. Ke, F. Y. Huang,"Verification of OnLine Vehicle Collision Avoidance Warning System using DSRC," World Academy of Science, Engineering and Technology, Vol. 55, pp 377-383, 2009.

[7] "DSRC Implementation Guide. A Guide to Users of SAE J2735 Message Sets over DSRC,"SAE International, 2010. 